\title{
Tethered Swimming Can Be Used to Evaluate Force Contribution for Short-Distance Swimiming Performance
}

\author{
Pedro G. Morouço, ${ }^{1,2}$ Daniel A. Marinho, ${ }^{2,3}$ Kari L. Keskinen, ${ }^{4}$ Juan J. Badillo, ${ }^{5}$ and \\ Mário C. Marques ${ }^{2,3}$ \\ ${ }^{1}$ Center for Rapid and Sustainable Product Development, Polytechnic Institute of Leiria, Leiria, Portugal; ${ }^{2}$ Research Center in \\ Sports, Health and Human Development, Covilhã, Portugal; ${ }^{3}$ Department of Sport Sciences, University of Beira Interior, \\ Covilhã, Portugal; ${ }^{4}$ Finnish Society of Sport Sciences, Helsinki, Finland; and ${ }^{5}$ Department of Sports and Informatics, University \\ of Pablo de Olavide, Seville, Spain
}

\begin{abstract}
Morouço, PG, Marinho, DA, Keskinen, KL, Badillo, JJ, and Marques, MC. Tethered swimming can be used to evaluate force contribution for short-distance swimming performance. $J$ Strength Cond Res 28(11): 3093-3099, 2014-The purpose of this study was two-fold: (a) to compare stroke and the physiological responses between maximal tethered and free front crawl swimming and (b) to evaluate the contribution of force exertion for swimming performance over short distances. A total of 34 male swimmers, representing various levels of competitive performance, participated in this study. Each participant was tested in both a 30 -second maximal tethered swimming test and a 50-m free swimming test. The tethered force parameters, the swimming speed, stroke (stroke rate [SR]), and the physiological responses (increase in blood lactate concentration $[\Delta \mathrm{BLa}]$, heart rate, and rate of perceived exertion) were recorded and calculated. The results showed no differences in stroke and the physiological responses between tethered and free swimming, with a high level of agreement for the $\mathrm{SR}$ and $\triangle \mathrm{BLa}$. A strong correlation was obtained between the maximum impulse of force per stroke and the speed $(r=0.91 ; p<0.001)$. Multiple regression analysis revealed that the maximum impulse and $S R$ in the tethered condition explained $84 \%$ of the free swimming performance. The relationship between the swimming speed and maximum force tended to be nonlinear, whereas linear relationships were observed with the maximum impulse. This study demonstrates that tethered swimming does not significantly alter stroke and the physiological responses compared with free swimming, and that the maximum impulse per stroke should be used to
\end{abstract}

Address correspondence to Pedro G. Morouço, pedro.morouco@ ipleiria.pt.

28(11)/3093-3099

Journal of Strength and Conditioning Research

(C) 2014 National Strength and Conditioning Association evaluate the balance between force and the ability to effectively apply force during sprint swimming. Consequently, coaches can rely on tethered forces to identify strength deficits and improve swimming performance over short distances.

KEY WoRDS exercise testing, sprint performance, stroke performance, impulse of force

\section{INTRODUCTION}

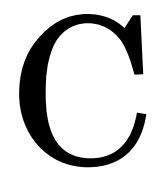

ompetitive swimming performance is determined by multifactorial parameters, including muscular force production while stroking (17), swimming technique (3), and aerobic/anaerobic energy production (25). The influence of force production on swimming performance has long been discussed (33) and suggests that the force exerted in water is a major factor for success $(2,10)$. For that reason, the magnitude of these forces has been the topic of many studies, although the complexity of the aquatic environment makes its assessment challenging (1). One possible method is to use a load-cell to register the tethered forces exerted while swimming. Indeed, tethered swimming has been shown to be a valid and reliable tool $(12,18)$, with muscular activity (5) and oxygen consumption (20) similar to those seen in free swimming, although minor kinematic changes have also been observed (21). Few studies have examined stroke and the physiological responses for both conditions, however.

In theory, the maximum tethered force corresponds to the propelling force that a swimmer must produce to overcome water resistance at the maximum free swimming speed $(15,23)$. Nevertheless, experiments involving tethered swimming have examined a variety of force parameters. For example, sprint speed has been found to be significantly correlated with the maximum force (17) or average of maximum force (33). Still, Taylor et al. (29) found that only the average force was a reliable parameter to estimate swimming performance in swimmers grouped by age. This incongruous use of parameters demonstrates that more studies are required to better understand this topic. Considering that 
propulsion occurs during the entire propulsive phase of the stroke cycle $(22,27)$, the effect of force with respect to time should be considered as follows:

$$
I=\int_{t_{1}}^{t_{2}} F \cdot d t
$$

where $I$ is the impulse of force, and $F$ is the applied force from time $t_{1}$ to $t_{2}$. Consequently, calculations of the impulse of force may be more accurate when interpreting the tethered forces (12), as the impulse of force depends on the magnitude, duration, and direction of the applied force. In addition, measures combining speed and force may be more strongly related to performance than are the peak values of force (19).

Assuming that tethered swimming can serve as a useful tool for force evaluation, the relationship between tethered force and swimming speed has generally been assumed to be linear $(23,33)$. However, Keskinen et al. (17) observed a positive nonlinear relationship between maximum force and maximum velocity. These authors stated that along with an increase in speed, there is a decline in force production at high velocities that follows the general force-velocity relationship. Variability in the swimming velocity, therefore, may not indicate variability in the stroking force. It is essential to further understand the relationships between these 2 elements, specifically for performance enhancement during sprint events. Although it is plausible that an association exists, the nature and strength of this relationship remain unclear. Additionally, research on the associations between strength variables and performance should overcome the limitations of previous correlational studies, such as the presentation of only zero-order (Pearson's) correlations and the inclusion of small sample sizes (often fewer than 20 subjects) (19).

Therefore, the aim of this study was two-fold: (a) to compare stroke (stroke rate $[\mathrm{SR}]$ ) and several physiological responses (blood lactate concentration, heart rate [HR], and rate of perceived exertion [RPE]) between tethered and free swimming and (b) to identify possible associations between tethered force parameters (maximum, average, and impulse) and the front crawl swimming speed. Based on the existing literature and the responses observed while tethering a swimmer, we considered the following hypotheses: (a) tethered and free swimming both result in a similar stroke and similar physiological responses, if a similar duration and intensity are used and (b) force exertion in a tethered condition is a major contributor to swimming performance over short distances. A better understanding of the relationships between the forces exerted in water and swimming performance may help coaches to identify swimmers with strength deficits.

\section{Methods}

\section{Experimental Approach to the Problem}

The main purposes of this study were to compare tethered and free swimming in terms of the SR and physiological responses and to analyze the role of force in short-distance swimming performance. To confirm the postulated hypotheses, stroke and physiological responses were both analyzed during tethered and free swimming tests performed on separate days. The tests were performed at the same time of the day to avoid any effect of circadian rhythms. The tethered swimming apparatus provided horizontal resistance so that the swimmer remained stationary in the water, and a load-cell was used to continuously record force exertion. The free swimming bouts were performed with an underwater start to diminish the effect of start and glide. The experiment was performed during the competitive period of the spring training cycle, thus ensuring that the subjects were in a prime training period. A sample size of 34 swimmers was chosen to allow for the incorporation of more variables and to assure more reasonable generalizability (19). The independent variables were the test conditions (tethered vs. free). The dependent variables were the maximum and average force, the maximum and average impulse of force, the SR, the increment of blood lactate concentration, the maximum $H R$, and the RPE.

\section{Subjects}

The study included 34 male competitive swimmers (age: $17.2 \pm 2.72$ years; range: $14.3-23.6$ years; height: $1.76 \pm 0.09$ $\mathrm{m}$; body mass: $67.4 \pm 9.94 \mathrm{~kg}$ ) representing various levels of competitive performance in $100-\mathrm{m}$ long course freestyle swimming (58.39 \pm 2.19 seconds; range: $52.1-63.0$ seconds). The swimmers had at least 5 years of experience in competitive swimming and have participated in national and international competitions. The study was conducted according to ethical standards in sport and exercise science research (14), and the protocol was fully approved by the Human Research Ethics Committee before any assessments were collected. Before participation, the approved procedures, risks, and benefits were explained to all subjects. The swimmers and their parents gave informed consent as part of their sport requirements, which is consistent with institutional policies for use of human subject research. Participants were excluded if they reported any injury or restriction within the last 3 months that hindered their performance.

\section{Procedures}

Swimmers randomly performed the tethered and free swimming tests 24 hours apart, ensuring that both conditions were assessed at the same time of day. Preceding each test, a 1,000-m moderate intensity warm-up (400-m swim, 100 -m pull, 100 -m kick, $4 \times 50 \mathrm{~m}$ at increasing speed, 200-m easy swim) was completed in a 50-m indoor swimming pool (water temperature of $26-27^{\circ} \mathrm{C}$ ).

The tethered swimming test consisted of a 30 -second front crawl maximal bout. A belt was attached to the hip with a $3.5-\mathrm{m}$ length of steel cable (Figure 1). The measuring device was a load-cell system connected to the swimmer, recording at $100 \mathrm{~Hz}$ with a measurement capacity of $4,905 \mathrm{~N}$. Data were exported to a laptop through an 


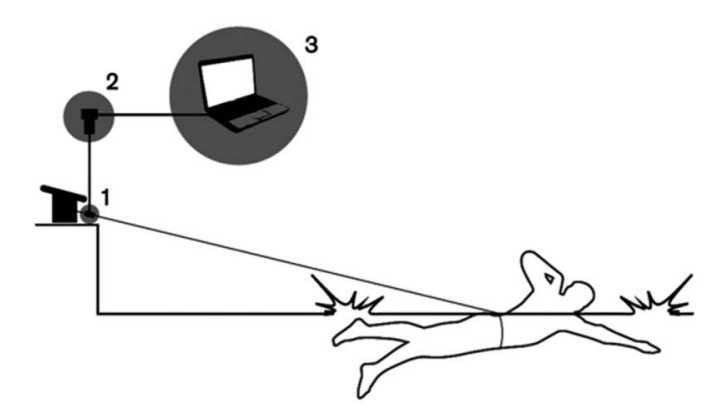

Figure 1. Apparatus used for the tethered swimming test: $1=$ load-cell; $2=$ ergometer data acquisition system; $3=$ personal computer.

ergometer data acquisition system (Globus, Codognè, Italy). Reliability and validity studies were conducted before the actual testing $(n=8)$. The participants underwent familiarization trials of the tethered testing procedure before data collection. The free swimming condition was evaluated with a 50-m front crawl maximal bout after an underwater start.

Swimmers were verbally encouraged throughout the tests to maintain maximal effort during the bouts.

\section{Measurements}

Tethered swimming data were exported to signal processing software (AcqKnowledge v.3.7; Biopac Systems, Santa Barbara, CA, USA) and filtered through a $4.5-\mathrm{Hz}$ cutoff low-pass filter. The cutoff value was chosen according to residual analysis (residual error vs. cutoff frequency). As the force vector in the tethered system presented a small angle to the horizontal, data were corrected by computing the horizontal component of the force (29), assessing the individual force-time curves. The following 4 parameters were estimated for each participant: (a) maximum force as the highest value obtained from the individual force-time curve, (b) average force as the mean of force values recorded during the 30 seconds, (c) maximum impulse (maxI) as the highest value of the impulse of force (equation 1) in a single-stroke, and (d) average impulse as the quotient of the sum of the single-stroke impulse and the number of strokes performed during the 30 -second tethered swim. Swimming speed was calculated from the time for the $50-\mathrm{m}$ (s50) free swim.

The SR $(\mathrm{Hz})$ was determined using a portable SR counter (Seiko, Tokyo, Japan) from 3 consecutive stroke cycles; for tethered swimming $\left(\mathrm{SR}_{\mathrm{TS}}\right)$, this sampling occurred at the middle of the test (at approximately 15 seconds), and for the 50-m $\left(\mathrm{SR}_{50}\right)$ free swim, it was in the midsection of the pool (approximately $25 \mathrm{~m}$ ). Blood lactate concentrations were determined from an earlobe blood sample taken just before each test and immediately after the test. The increase in blood lactate concentration $(\triangle \mathrm{BLa})$ was measured using a portable analyzer (Lactate Pro, Arkay, Japan). Heart rate was continuously recorded by a HR monitor worn by each subject (RS800CX, Polar Electro Oy, Kempele, Finland). The RPE was assessed verbally using the original Borg's scale with incremental descriptors of the perception of effort, ranging from 6 (no exertion at all) to 20 (maximal exertion) (7).

\section{Statistical Analyses}

For the validity and reliability studies, 8 swimmers completed 2 tethered swimming tests in consecutive days. The normality of all distributions was verified using KolmogorovSmirnov tests, and parametric statistical analysis was adopted. The magnitude of differences in SR, $\triangle B L a, H R$, and RPE between tethered and free swimming were evaluated with a paired-samples $t$-test. The effect sizes $(d)$ of the differences were calculated. Cohen's categories were used to evaluate the magnitude of these effect sizes (small if $0 \leq|d| \leq$ 0.5 , medium if $0.5<|d| \leq 0.8$, and large if $|d|>0.8)$ (8). Pearson's correlation coefficients $(r)$ were determined to assess the relationships among selected variables, and linear and nonlinear regression analyses were applied to evaluate the potential associations. Multiple regression analysis was used to verify the combination of significant variables that could explain performance variability in the free swimming. Limits of agreement between parameters measured in tethered and free swimming were derived following the recommendations by Bland and Altman (4), and intraclass correlation coefficients were used 

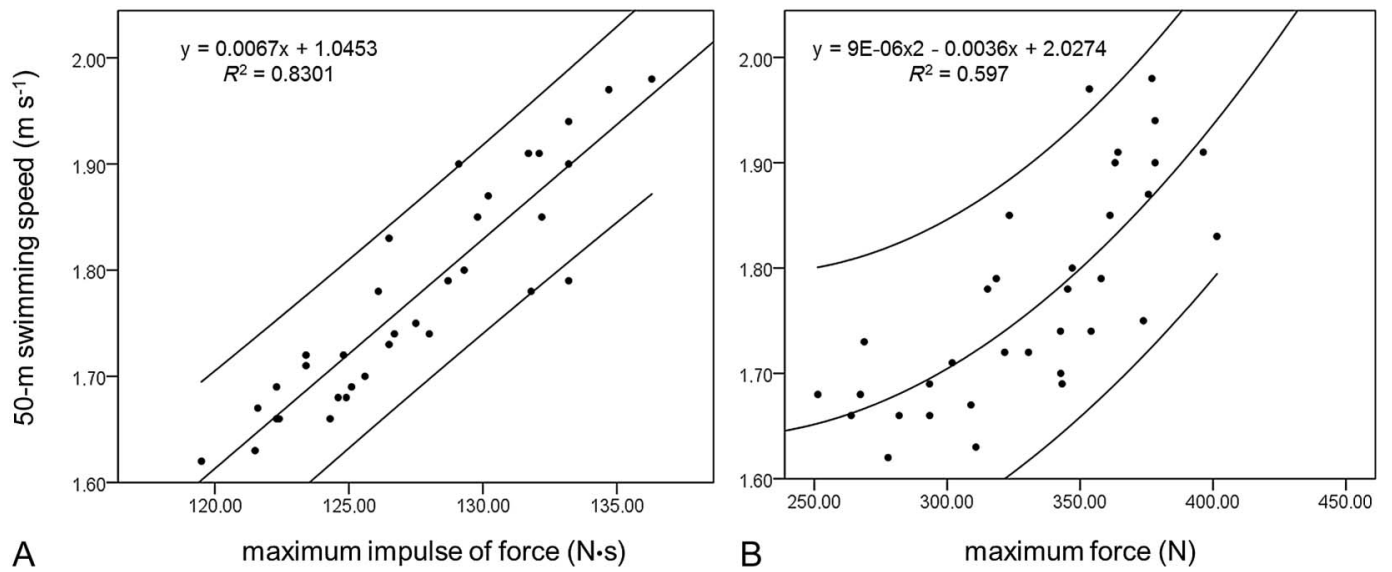

Figure 2. The scatter of plots together with the lines for best fit and $95 \%$ confidence interval of the relationship between the 50 - $m$ swimming speed and the maximum impulse of force $(A)$, and the maximum force $(B)$.

to assess the validity of procedures. All statistical procedures were performed using SPSS 20.0 (Chicago, IL, USA). The level of statistical significance was set at $p \leq 0.05$.

\section{RESUlts}

Table 1 includes descriptive statistics for the force parameters, the swimming speed, and the stroke and physiological responses for tethered and free swimming conditions. There were no differences in $\mathrm{SR}, \triangle \mathrm{BLa}, \mathrm{HR}$, or RPE within the 30second tethered swim test and the 50-m front crawl swim (time $=28.3 \pm 1.6$ seconds). Intraclass correlation coefficients were between $0.95(0.92-0.98)$ and $0.98(0.96-0.99)$ for the force measurements $(n=8)$.
There were significant relationships between the tethered parameters and free swimming performance. The free swimming speed (s50) was found to be positively correlated with both the maximum and average force $(r=0.76$ and $r=$ $0.81 ; p<0.001$, respectively) and the maximum and average impulse $(r=0.91$ and $r=0.70 ; p<0.001$, respectively). Moreover, the association between the free swimming speed tended to be linear at maximum impulse (Figure $2 \mathrm{~A}$ ) but nonlinear at maximum force (Figure 2B). Adding the $\mathrm{SR}_{\mathrm{TS}}$ with maxI into a multiple regression model, it was possible to explain $84 \%$ of the $50-\mathrm{m}$ performance variation $(\mathrm{t} 50(\mathrm{~s})=$ $71.404-0.335 \mathrm{SR}-0.336 \mathrm{maxI} ; R^{2}=0.84 ; \mathrm{R}_{\mathrm{a}}^{2}=0.83$; $p<0.001)$.
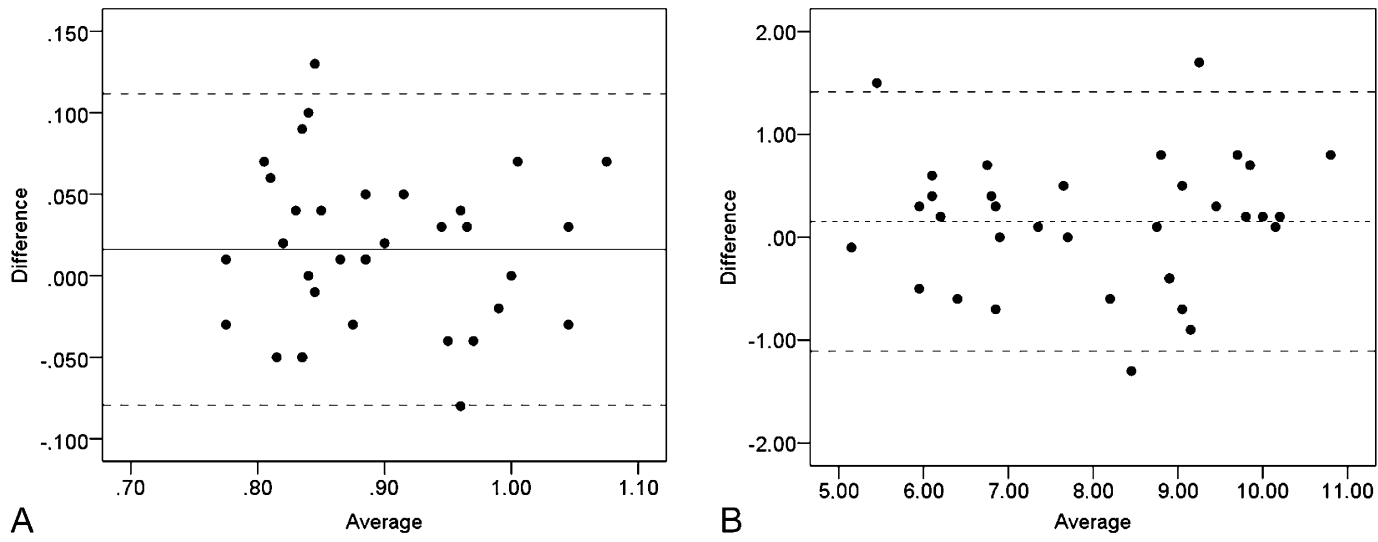

Figure 3. Bland and Altman plots of comparison between the tethered swimming and 50-m front crawl for the SR (A) and blood lactate concentration (B). Average difference line (solid line) and 95\% confidence interval (dashed lines) are indicated. SR $=$ stroke rate. 
Both SR and $\triangle \mathrm{BLa}$ assessed in tethered and free swimming were highly correlated within conditions $(r=0.84$ and $r=0.92 ; p<0.001$, respectively). Likewise, a moderate relationship was observed between $\Delta \mathrm{BLa}$ in tethered swimming and s50 $(r=0.55 ; p<0.01)$. Heart rate did not exhibit significant correlations with other variables. A strong correlation between RPE rated after tethered and after free swimming was observed $(r=0.72 ; p<0.001)$.

Comparing tethered and free swimming, the intraclass correlation coefficients were between $0.91(0.82-0.96)$ and $0.96(0.92-0.98)$ for SR and $\triangle B L a$, respectively (Figure 3 ). The average differences were rather low, with limits of agreement (average $\pm 1.96 S D$ ) ranging from -0.079 to 0.112 for $\mathrm{SR}$ and from -1.108 to 1.414 for $\Delta \mathrm{BLa}$.

\section{Discussion}

The purpose of this study was two-fold: (a) to compare stroke and physiological responses between tethered and free swimming and (b) to analyze the contribution of force exertion for swimming performance over short distances. Our results suggest that tethering the swimmer did not alter the stroke or any physiological responses compared with free swimming of similar duration and intensity. Moreover, the maximum impulse per stroke exhibits a high level of accuracy in predicting free swimming performance. Analyses of the tethered swimming parameters clarified the role of tethered forces, which can be used to improve swimming performance, in agreement with previously established hypotheses.

The stroke and physiological responses did not differ significantly between the 30 -second tethered test and $50-\mathrm{m}$ free swimming. This similarity suggests that tethered swimming can be used to evaluate swimmers, despite the minor kinematic differences that have been noted (21). Stroke rate should be evaluated from an individual perspective, as swimmers tend to use a freely chosen rate (16). This variable is informative with respect to the performance potential (26), and in this study, it showed a high level of agreement between conditions. Therefore, we can assume that 30 seconds of the tethered test closely replicated the effort of a 50-m maximal front crawl.

The energy contribution during swimming drills has long been the subject of interest for scientists and coaches (24) because this information is helpful when developing training plans (25). With that in mind, the concentration of blood lactate is commonly assessed (26), and its relationship with swimming speed is an important consideration for swimming performance enhancement (6). Blood lactate concentrations after the tethered test were similar to those obtained after free swimming. This finding contradicts the idea that to remain stationary, the swimmer relies mostly on fast glycolytic muscle fibers, thereby increasing the production and diffusion of lactic acid into the blood (32). Furthermore, similar responses were also observed for maximal $\mathrm{HR}$ and the RPE between tests. This latter parameter has been shown to be an effective measure of exercise intensity and has also been used to develop a swim training plan (30). Therefore, the results confirmed the hypothesis that a 30-second maximal tethered swimming is an accurate simulation of the performance in a 50-m maximal swim, regarding stroke and the physiological demands.

Tethered swimming has been proven to be a highly reliable methodology $(11,18)$ to obtain a feasible estimation of the propelling force necessary to overcome water resistance during free swimming (2). The magnitudes of the maximum forces identified in this study are in line with those found in previous studies. For example, Keskinen et al. (17) reported maximum forces lower than those of this study, as was expected, because the authors used an elastic cord attached to the swimmer. Using a nonelastic cable, Sidney et al. (28) found higher values of maximum force due to the higher performance capacity of those swimmers, as confirmed by their faster swimming speeds. Moreover, various experiments with tethered swimming have used varying duration bouts, and most experiments involved tests that lasted less than 30 seconds. Considering that the average force depends on the duration of testing, the higher outputs reported in those experiments are expected (33). For this study, 30-second maximal tethered swimming was used to simulate the time duration of the $50-\mathrm{m}$ maximal front crawl free swimming (a typical distance in training series and often used to set high-intensity training bouts), and no differences $(p<0.001)$ were observed between the durations of the 2 tests.

Considering that propulsion can occur throughout the propulsive phase of the stroke $(22,27)$, the impulse of force per stroke was calculated, and the maximum and average values were registered. Similarly, Dopsaj et al. (12) conducted an experiment that included a 20 -second maximal tethered swimming and assessed the average values of impulse incorporated within 5-, 10-, 15-, and 20-second intervals, which were higher than the results of the current study, due to the extended duration of time. Nonetheless, these authors indicated that when tethered swimming is used for top swimmers, the impulse of force should be considered. Indeed, the maximum force includes information concerning a single point per stroke cycle, which is when the maximum force is reached. However, lower force levels can be maintained during longer lasting strokes, which may produce similar (or even higher) momentum changes than a higher force applied over a shorter time (13). This concept was confirmed by the present data, given that the multiple regression model with maximum impulse and $\mathrm{SR}$ in tethered swimming explained $84 \%$ of the 50 -m free swimming performance. Theoretically, during tethered swimming, these 2 variables should constitute the work performed against the force transducer and consequently explain $100 \%$ of the tethered swimming performance. Because the difference between tethered swimming and free swimming is water resistance, the present results suggest that $16 \%$ of the variance is due to the active drag that the swimmer must overcome during free swimming. 
Since the study of Yeater et al. (33), several investigations have demonstrated a strong relationship between tethered forces and swimming speed $(12,17)$. This relationship differs according to age and maturity (31), status of competitive performance (28), and swimming distance (23). Nevertheless, most of the aforementioned studies confirmed the relationship of tethered forces with swimming speed over noncompetitive distances (e.g., $10 \mathrm{~m}$ ). When evaluating swimmers, using competitive distances can aid the possible transfer to a swim training plan (13). The 50 -m freestyle uses high intensities during a short period of time. Thereby, studies aiming to clarify the role of stroking force in those bouts may clarify the contribution of strength for swimming performance over short distances. First, a very strong relationship between swimming speed and maximum impulse of force was observed, suggesting that the impulse of force is more appropriate to describe the performance of sprinters over short distances (12), and second, blood lactate concentrations after free swimming exhibited moderate-to-strong relationships with the tethered force parameters, corroborating previous results (24). These findings emphasize the conclusion that the demands of high-intensity front crawl swimming seem to be strongly influenced by the effective application of force during the arm stroke (9).

Taking into consideration the maximum force (the most common variable assessed during tethered swimming), a nonlinear relationship with $50-\mathrm{m}$ swimming speed was observed (Figure 2B). This nonlinear association suggests that studies using this variable underestimated the role of stroke force mechanics in swimming performance. This idea was previously reported (17), indicating that a limit in the force to increase swimming speed necessarily occurs. Consequently, impulse of force should be considered. That is, experiments should not only focus on the magnitude of the exerted forces but also take into consideration the time spectrum of their application. During tethered swimming, force production represents the magnitude of the performed pull drive, and as such, it represents the working potential to be realized during free swimming (12).

In conclusion, we found that tethered and free swimming of similar duration and intensity exhibit the same responses in stroke and physiological variables. Therefore, tethered swimming testing may be used to analyze the balance between force and the ability to effectively apply force during high-speed swimming. The impulse of force when assessing forces per stroke should be used as a determining element for explaining swimming performance. In other words, the maximum force does not completely reflect the stroke mechanics of swimming performance, especially at high swimming speeds.

\section{Practical Applications}

First, this study demonstrates that tethered swimming produces a similar stroke and similar physiological responses compared with free swimming of the same duration and intensity. From a practical perspective, these results clearly highlight that tethered swimming can be used as a specific evaluation tool to assess swimmers. Second, force exertion is a markedly significant factor determining swimming performance over short distances. A regular assessment of the exerted tethered forces may highlight whether a swimmer is developing force but not the ability to effectively apply that force (e.g., if the swimmer increases the maximum tethered force but not the swimming speed). In those cases, coaches should pay closer attention to the swimmer's technique rather than to improvements in strength. Finally, when evaluating top swimmers, the impulse of force must be considered. Our data indicated that lower maximum forces were able to produce a higher impulse, and consequently, a better explanation of free swimming sprint distance performance. Further investigations should focus on a variety of exercise durations to better understand potential applications to other competitive swimming events.

\section{ACKNowledgments}

The authors thank all swimmers and coaches who participated in this research. This investigation was supported by grants of the Portuguese Science and Technology Foundation (SFRH/BD/66910/2009) and University of Beira Interior (UBI/FCSH/Santander/2010). The authors do not have professional relationships with companies or manufacturers who will benefit from the results of this study. The results of this study do not constitute endorsement of the product by the authors or the National Strength and Conditioning Association.

\section{REFERENCES}

1. Akis, T and Orcan, Y. Experimental and analytical investigation of the mechanics of crawl stroke swimming. Mech Res Comm 31: 243-261, 2004.

2. Barbosa, AC, Castro, FS, Dopsaj, M, Cunha, SA, and Júnior, OA. Acute responses of biomechanical parameters to different sizes of hand paddles in front-crawl stroke. J Sports Sci 31: 1015-1023, 2013.

3. Barbosa, TM, Bragada, JA, Reis, VM, Marinho, DA, Carvalho, C, and Silva, AJ. Energetics and biomechanics as determining factors of swimming performance: Updating the state of the art. J Sci Med Sport 13: 262-269, 2010.

4. Bland, JM and Altman, DG. Applying the right statistics: Analyses of measurement studies. Ultrasound Obstet Gynecol 22: 85-93, 2003.

5. Bollens, E, Annemans, L, Vaes, W, and Clarys, JP. Peripheral EMG comparison between fully tethered and free front crawl swimming. In: Swimming Science $V$. B.E. Ungerechts, K. Wilke, and K. Reischle, eds. London, United Kingdom: Spon Press, 1988. pp. 173-181.

6. Bonifazi, M, Martelli, G, Marugo, L, Sarella, F, and Carli, G. Blood lactate accumulation in top level swimmers following competition. J Sports Med Phys Fit 33: 13-18, 1993.

7. Borg, G. Borg's Perceived Exertion and Pain Scales. Champaign, IL: Human Kinetics, 1988.

8. Cohen, J. Statistical Power Analysis for the Behavioural Sciences. Hillsdale, NJ: Lawrence Erlbaum Associates, 1988.

9. Costill, DL, Kovaleski, J, Porter, D, Kirwan, J, Fielding, R, and King, D. Energy expenditure during front crawl swimming: Predicting success in middle-distance events. Int J Sports Med 6: 266-270, 1985. 
10. Dominguez-Castells, R, Izquierdo, M, and Arellano, R. An updated protocol to assess arm swimming power in front crawl. Int $J$ Sports Med 34: 324-329, 2012.

11. Dopsaj, M, Matković, I, Thanopoulos, V, and Okicić, T. Reliability and validity of basic kinematics and mechanical characteristics of pulling force in swimmers measured by the method of tethered swimming with maximum intensity of 60 seconds. Phys Educ Sport 1: 11-22, 2003.

12. Dopsaj, M, Matković, I, and Zdravković, I. The relationship between $50 \mathrm{~m}$-Freestyle results and characteristics of tethered forces in male sprinters: A new approach to tethered swimming test. Phys Educ Sport 1: 15-22, 2000.

13. Formosa, DP, Mason, B, and Burkett, B. The force-time profile of elite front crawl swimmers. J Sports Sci 29: 811-819, 2011.

14. Harris, DJ and Atkinson, G. Update-Ethical standards in sport and exercise science research. Int J Sports Med 32: 819-821, 2011.

15. Keskinen, KL. Evaluation of technique performances in freestyle swimming. Kines 2: 30-38, 1997.

16. Keskinen, KL and Komi, PV. Stroking characteristics of front crawl swimming during exercise. J Appl Biomech 9: 219-226, 1993.

17. Keskinen, KL, Tilli, LJ, and Komi, PV. Maximum velocity swimming: Interrelationships of stroking characteristics, force production and anthropometric variables. Scand J Med Sci Sport 11: 87-92, 1989.

18. Kjendlie, PL and Thorsvald, K. A tethered swimming power test is highly reliable. Port J Sport Sci 6: 231-233, 2006.

19. Knudson, DV. Correcting the use of the term "power" in the strength and conditioning literature. J Strength Cond Res 23: 1902-1908, 2009.

20. Lavoie, JM and Montpetit, RR. Applied physiology of swimming. Sport Med 3: 165-189, 1986.

21. Maglischo, C, Maglischo, E, Sharp, R, Zier, D, and Katz, A. Tethered and nontethered crawl swimming. In: Proceedings of ISBSSports Biomechanics. J. Terauds, K. Barthels, E. Kreighbaum, R. Mann, and J. Crakes, eds. Del Mar, CA: Academic Publication, 1984. pp. 163-176.

22. Marinho, DA, Silva, AJ, Reis, VM, Barbosa, TM, Vilas-Boas, JP, Alves, FB, Machado, L, and Rouboa, AI. Three-dimensional CFD analysis of the hand and forearm in swimming. J Appl Biomech 27: 74-80, 2011.
23. Morouço, PG, Keskinen, KL, Vilas-Boas, JP, and Fernandes, RJ. Relationship between tethered forces and the four swimming techniques performance. J Appl Biomech 27: 161-169, 2011.

24. Morouço, PG, Vilas-Boas, JP, and Fernandes, RJ. Evaluation of adolescent swimmers through a 30-s tethered test. Ped Exerc Sci 24: 312-321, 2012.

25. Peyrebrune, MC, Toubekis, AG, Lakomy, HKA, and Nevill, ME. Estimating the energy contribution during single and repeated sprint swimming. Scand J Med Sci Sports 2012. doi: 10.1111/j.16000838.2012.01517.x.

26. Psycharakis, SG, Cooke, CB, Paradisis, GP, O'Hara, J, and Phillips, G. Analysis of selected kinematical and physiological performance determinants during incremental testing in elite swimmers. J Strength Cond Res 22: 951-957, 2008.

27. Schleihauf, RE. A Hydrodynamic analysis of swimming propulsion. In: Szoimming III-International Series of Sports Sciences. J. Terauds and E. Bedingfield, eds. Baltimore, MD: University Park Press, 1979. pp. 70-109.

28. Sidney, M, Pelayo, P, and Robert, A. Tethered forces in crawl stroke and their relationship to anthropometrics characteristics and sprint swimming performance. J Hum Mov Stu 31: 1-12, 1996.

29. Taylor, S, Lees, A, Stratton, G, and MacLaren, D. Reliability of force production in tethered freestyle swimming among competitive agegroup swimmers. J Sports Sci 19: 12-13, 2001.

30. Ueda, T and Kurokawa, T. Relationships between perceived exertion and physiological variables during swimming. Int J Sports Med 16: 385-389, 1995.

31. Vorontsov, A, Dyrco, V, Binevsky, D, Solomatin, V, and Sidorov, N. Patterns of growth for some characteristics of physical development, functional and motor abilities in boy-swimmers 11-18 years. In: Biomechanics and Medicine in Swimming VIII. K. Keskinen, P. Komi, and A. Hollander, eds. Jyväskulä, Finland: Gummerus Printing, 1999. pp. 327-335.

32. West, SA, Drummond, MJ, Vanness, JM, and Ciccolella, ME. Blood lactate and metabolic responses to controlled frequency breathing during graded swimming. J Strength Cond Res 19: 772-776, 2005.

33. Yeater, RA, Martin, RB, White, MK, and Gilson, KH. Tethered swimming forces in the crawl, breast and back strokes and their relationship to competitive performance. J Biomech 14: 527-537, 1981. 